\title{
Phase Synchronization in Coupled Sprott Chaotic Systems Presented by Fractional Differential Equations
}

\author{
G. H. Erjaee ${ }^{1,2}$ and M. Alnasr ${ }^{1}$ \\ ${ }^{1}$ Mathematics Department, Qatar University, Doha, Qatar \\ ${ }^{2}$ Mathematics Department, Shiraz University, Shiraz, Iran \\ Correspondence should be addressed to G. H. Erjaee, erjaee@qu.edu.qa
}

Received 21 June 2009; Accepted 1 November 2009

Recommended by B. Sagar

Phase synchronization occurs whenever a linearized system describing the evolution of the difference between coupled chaotic systems has at least one eigenvalue with zero real part. We illustrate numerical phase synchronization results and stability analysis for some coupled Sprott chaotic systems presented by fractional differential equations.

Copyright (C) 2009 G. H. Erjaee and M. Alnasr. This is an open access article distributed under the Creative Commons Attribution License, which permits unrestricted use, distribution, and reproduction in any medium, provided the original work is properly cited.

\section{Introduction}

Phase synchronization has been reported for various coupled chaotic systems [1, 2]. This phenomenon occurs when the linearized system describing the evolution of the difference between a pair of chaotic systems has some zero or positive conditional Lyapunov exponents. As we have shown in [2], this behavior also depends upon the eigenvalues of the linearized difference system. More precisely, suppose that two identical chaotic systems $\dot{\mathbf{x}}=\mathbf{F}(\mathbf{x}(t))$ and $\dot{\mathbf{y}}=\mathbf{F}(\mathbf{y}(t))$ are coupled, as derive and response systems, according to the method of Pecora and Carroll [3] by a continuous coupling function $\mathbf{h}(\mathbf{x})$. If the system $\dot{\mathbf{e}}=\mathbf{F}(\mathbf{x}, \mathbf{h})-$ $\mathbf{F}(\mathbf{y}, \mathbf{h})=\mathbf{F}(\mathbf{x}, \mathbf{h})-\mathbf{F}(\mathbf{x}+\mathbf{e}, \mathbf{h})$, which described the evolution of the difference between two identical systems, has a zero or constant solutions, then the two systems have complete synchronization or phase synchronization, recursively $[2,4-6]$. Indeed, an analysis of the linearized difference system, $\dot{\mathbf{e}}=\mathbf{A e}$, may yield considerable information about the dynamics of the coupled chaotic systems. For the synchronization, we need to determine the conditional Lyapunov exponents of this system, and for the phase synchronization we need to also find the eigenvalues of the system [2]. As shown below, similar results apply to the phase synchronization Sprott systems [7], presented by Fractional Differential Equations (FDEs). 
That is, the real parts of the eigenvalues of the evolution matrix A provide information about the ability to synchronize coupled chaotic systems presented by FDEs. In illustrated numerical results, we can see several cases that may arise between derive and response systems in the form of FDEs. In some cases, the difference between derive and response is constant, while in other cases it is periodic or a function of time. These and some other cases are presented in Section 2, followed by a stability discussion in Section 3.

\section{Coupled Sprott Chaotic Systems Presented by FDEs}

In this section we consider four different Sprott systems presented by FDEs. In each case the derive and response systems are coupled using the methods of Carroll and Pecora $[3,8]$.

Example 2.1. Consider the coupled Sprott-S systems presented by the FDEs:

$$
\begin{array}{ll}
D^{\alpha} x_{1}=-x_{1}+a x_{2} & D^{\beta} y_{1}=-y_{1}+a y_{2}, \\
D^{\alpha} x_{2}=x_{1}+x_{3}^{2} & D^{\beta} y_{2}=y_{1}+y_{3}^{2}, \\
D^{\alpha} x_{3}=1+x_{1} & D^{\beta} y_{3}=1+x_{1} .
\end{array}
$$

These systems are coupled through the third equation, where $D^{\alpha} \mathbf{x}(t)=J^{n-\alpha} D^{n} \mathbf{x}(t)$ is the $n$th order Riemann-Liouville integral operator defined by $J^{n} \mathbf{x}(t)=(1 / \Gamma(n)) \int_{0}^{t}(t-\tau)^{n-1} \mathbf{x}(\tau) d \tau$, with $0<\alpha \leq 1$ and $D^{n}(\cdot)$ being ordinary derivative of order $n$ for time $t>0$. By the GrunwaldLetnikov method $[9,10]$ the fractional derivative is discretized as $D^{\alpha} \mathbf{x}(t)=\sum_{k=0}^{\left[t_{n} / h\right]} c_{k}^{\alpha} \mathbf{x}\left(t_{n-k}\right)$. Here, $h$ is the step size, $\left[t_{n} / h\right]$ denotes the integer part of $t_{n} / h, t_{n}=n h$, and $c_{k}^{\alpha}$ are the Grunwald-Letnikov coefficients defined by $c_{k}^{\alpha}=h^{-\alpha}(-1)^{k}\left(\begin{array}{l}\alpha \\ k\end{array}\right), k=0,1,2, \ldots$ These coefficients can also be evaluated, recursively, by $c_{0}^{\alpha}=h^{-\alpha}$ and $c_{k}^{\alpha}=(1-(1+\alpha) / k) c_{k-1}^{\alpha}, k=1,2,3, \ldots$ Using these definitions, the above coupled Sprott-S systems are discretized as follows:

$$
\begin{aligned}
& x_{1}\left(t_{n}\right)=h^{\alpha}\left[-x_{1}\left(t_{n-1}\right)+a x_{2}\left(t_{n-1}\right)\right]-\sum_{k=1}^{N}\left(1-\frac{1+\alpha}{k}\right) x_{1}\left(t_{n-k}\right), \\
& x_{2}\left(t_{n}\right)=h^{\alpha}\left[x_{1}\left(t_{n-1}\right)+x_{3}^{2}\left(t_{n-1}\right)\right]-\sum_{k=1}^{N}\left(1-\frac{1+\alpha}{k}\right) x_{2}\left(t_{n-k}\right), \\
& x_{3}\left(t_{n}\right)=h^{\alpha}\left[1+x_{1}\left(t_{n-1}\right)\right]-\sum_{k=1}^{N}\left(1-\frac{1+\alpha}{k}\right) x_{3}\left(t_{n-k}\right), \\
& y_{1}\left(t_{n}\right)=h^{\beta}\left[-y_{1}\left(t_{n-1}\right)+a y_{2}\left(t_{n-1}\right)\right]-\sum_{k=1}^{N}\left(1-\frac{1+\beta}{k}\right) y_{1}\left(t_{n-k}\right), \\
& y_{2}\left(t_{n}\right)=h^{\beta}\left[y_{1}\left(t_{n-1}\right)+y_{3}^{2}\left(t_{n-1}\right)\right]-\sum_{k=1}^{N}\left(1-\frac{1+\beta}{k}\right) y_{2}\left(t_{n-k}\right), \\
& y_{3}\left(t_{n}\right)=h^{\beta}\left[1+x_{1}\left(t_{n-1}\right)\right]-\sum_{k=1}^{N}\left(1-\frac{1+\beta}{k}\right) y_{3}\left(t_{n-k}\right) .
\end{aligned}
$$




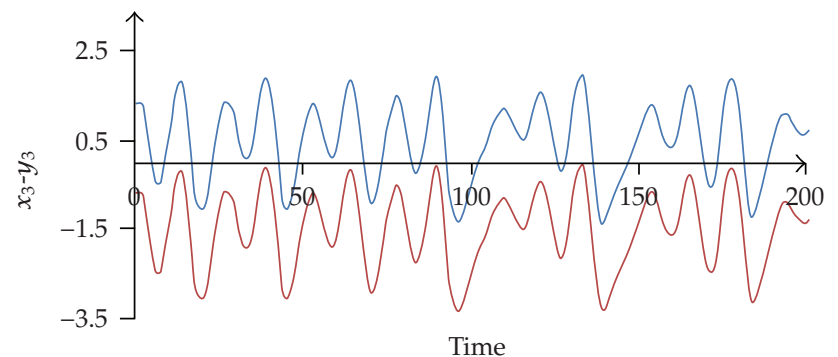

(a)

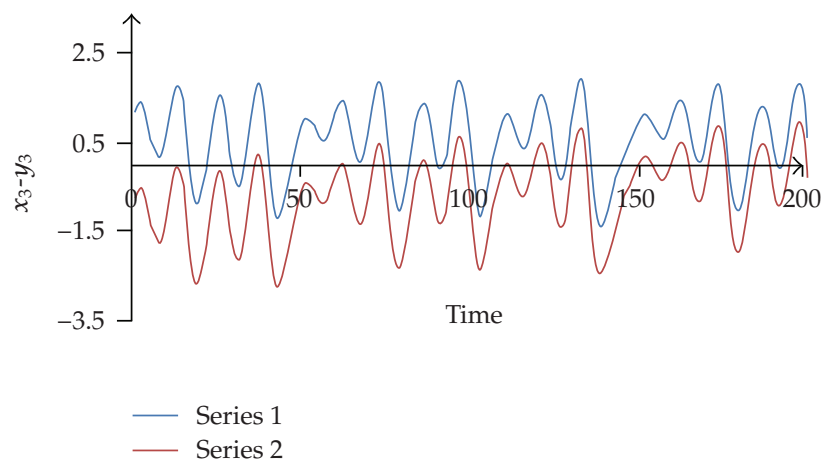

(b)

Figure 1: Phase synchronization for coupled chaotic Sprott-S systems presented by FDEs. (a) shows the constant difference between the derive (Series 1) and response (Series 2) systems for $\alpha=\beta=1$ and (b) for $\alpha=\beta=0.96$.

Numerical chaotic results for $a=-4$ in the $\left(x_{3}, t\right)$ and $\left(y_{3}, t\right)$ planes are illustrated in Figure 1. Figure 1(a) shows the phase synchronization for $\alpha=\beta=1$, which is in complete agreement with the direct Euler solutions of the original system for Sprott-S ODEs with $h=5 \times 10^{-4}$, and Figure 1(b) shows the phase synchronization for $\alpha=\beta=0.96$ with the same value of $h$. As we can see in both figures, the trajectories of the derive and response show that the response attractor is a copy of the derive displaced by some distance in the $y$ direction. This distance depends on the initial conditions. It is easy to see that the evolution matrix $\mathbf{A}$ in above Sprott-S systems of FDEs takes the form $\left(\begin{array}{ccc}-1 & -4 & 0 \\ 1 & 0 & 2 e_{3} \\ 0 & 0 & 0\end{array}\right)$ which has obviously a zero and two complex eigenvalues $-1 / 2 \pm i \sqrt{15} / 2$ around $\mathbf{e}=\mathbf{0}$. So we should expect the phase synchronization only between $x_{3}$ and $y_{3}$.

Example 2.2. In this example we consider two Sprott-C systems that are coupled by the second method of Pecora and Carroll. That is, $y_{1}$ variable in the response system is completely replaced by its counterpart $x_{1}$ variable in the derive system,

$$
\begin{array}{ll}
D^{\alpha} x_{1}=x_{2} x_{3}, & D^{\beta} y_{2}=x_{1}-y_{2}, \\
D^{\alpha} x_{2}=x_{1}-x_{2}, & D^{\beta} y_{3}=1+a x_{1}^{2} . \\
D^{\alpha} x_{3}=1+a x_{1}^{2}, &
\end{array}
$$




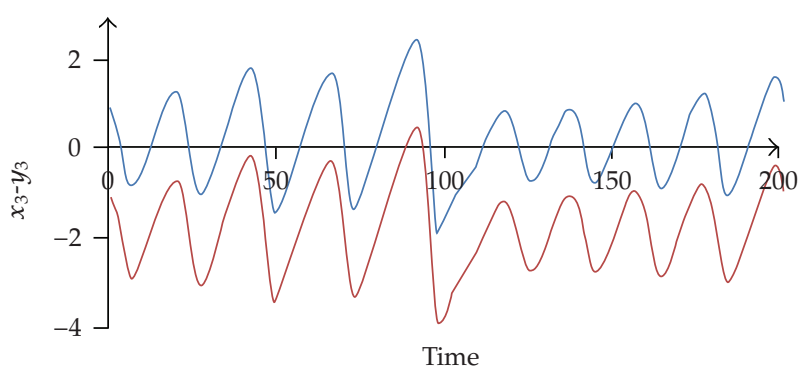

(a)

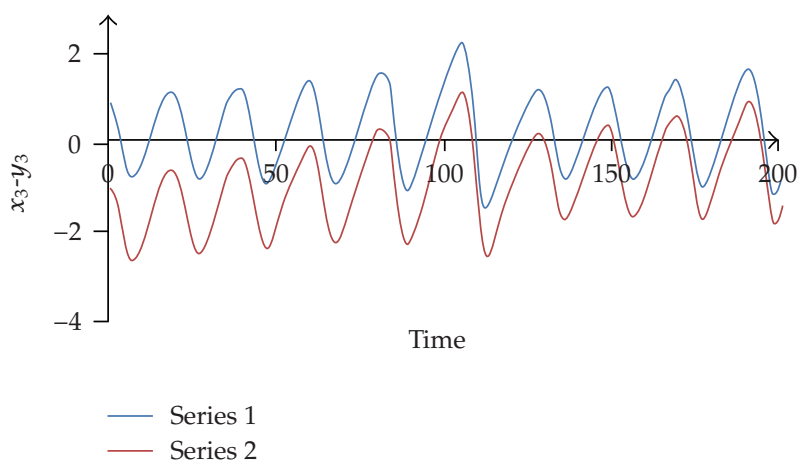

(b)

Figure 2: Phase synchronization for coupled chaotic Sprott-C presented by FDEs. (a) shows the constant differnce between the derive (Series 1) and response (Series 2) systems for $\alpha=\beta=1$ and (b) for $\alpha=\beta=$ 0.96 .

In this example, for the chaotic case $a=-1$, the eigenvalues of the evolution matrix $\mathbf{A}$ are -1 and zero, and hence, we expect phase synchronization between $x_{3}$ and $y_{3}$. The numerical results from the related discretized system are illustrated in Figure 2. Figure 2(a) shows the phase synchronization between $x_{3}$ and $y_{3}$ for $\alpha=\beta=1$ and $h=5 \times 10^{-5}$ that are in complete harmony with the numerical results found by Euler's method for the coupled Sprott-C presented by ODEs. Figure 2(b) shows the phase synchronization between $x_{3}$ and $y_{3}$ for $\alpha=\beta=0.96$ and $h=5 \times 10^{-5}$. The difference between the derive and response in these two cases converges to a constant depending on the initial values. Indeed, as long as the phase synchronization exists, this difference between derive and response systems will remain constant for any values of $\alpha$ and $\beta$. In this case we should note that, since there is only one negative eigenvalue, the phase synchronization is very sensitive to the values of $\alpha$, $\beta$ as well as to the initial conditions. That is, a slight change in these values may replace the chaotic behavior with periodic or steady state solutions.

Example 2.3. Next, consider two Sprott-L systems linked through the second Pecora-Carroll method:

$$
\begin{array}{ll}
D^{\alpha} x_{1}=x_{2}+a x_{3}, & D^{\beta} y_{2}=x_{2}+a y_{3}, \\
D^{\alpha} x_{2}=b x_{1}^{2}-x_{2}, & D^{\beta} y_{3}=1-y_{2} . \\
D^{\alpha} x_{3}=1-x_{1}, &
\end{array}
$$


The Sprott-L system presented by ODEs with the parameters $a=3.9$ and $b=0.9$ is chaotic, and in its above coupled form, the related evolution matrix $\mathbf{A}$ has two imaginary eigenvalues $\lambda_{1,2}= \pm \sqrt{3.9}$. In this case, as illustrated in Figure 3, phase synchronization between the derive and response occurs in such way that the differences between them will change in oscillatory fashion for different values of $\alpha$ and $\beta$. The frequency of this oscillation depends on the imaginary part of the eigenvalues, but its amplitude is constant depending on the initial values. This phenomenon is called marginal oscillatory synchronization [11]. Figure 3 shows solutions for $x_{1}, y_{1}$ and their differences for various values of $\alpha$ and $\beta$. As illustrated in Figure 3(d), the difference between derive and response for the values $\alpha=\beta=0.96$ is converging to zero in an oscillatory fashion. From numerical results, we note that this coupled system is no longer chaotic for values of $\alpha$ and $\beta$ less than 0.96 .

Example 2.4. Finally, couple two Sprott-R systems presented by FDEs by the first method of Pecora-Carroll as follows:

$$
\begin{array}{ll}
D^{\alpha} x_{1}=a-x_{2}, & D^{\alpha} y_{1}=a-y_{2}, \\
D^{\alpha} x_{2}=b+x_{3}, & D^{\alpha} y_{2}=b+x_{3}, \\
D^{\alpha} x_{3}=x_{1} x_{2}-x_{3}, & D^{\alpha} y_{3}=y_{1} y_{2}-y_{3} .
\end{array}
$$

This Sprott-R system is chaotic for $a=0.9$ and $b=0.4$. Here the related evolution matrix $\mathbf{A}$ has two zero eigenvalues. In this case, note that for $\alpha=\beta=1$, we get $\dot{e}_{1}=y_{2}-x_{2}$ from the first equations in the derive and response systems. On the other hand, it is clear that from the second equations that $y_{2}-x_{2}=c$. This means that $\dot{e}_{1}=c$, so $e_{1}=c t$, and the difference between $x_{1}$ and $y_{1}$ is a straight line with slope equal to $c$, while the difference between $x_{2}$ and $y_{2}$ remains constant. As illustrated in Figure 4, this is also the case for values of $\alpha$ and $\beta$ less than one.

Here, as with the examples above, the behavior of the coupled systems presented by FDEs is not chaotic for values $\alpha$ and $\beta$ less than 0.96. For example, Figure 5 shows the solutions of coupled Sprott-R systems for $\alpha=\beta=0.93$ for which chaotic solutions become periodic solutions.

\section{Convergence Criteria}

Suppose two identical chaotic FDEs $\mathbf{D}^{\alpha} \mathbf{x}=\mathbf{F}(\mathbf{x}(t))$ and $\mathbf{D}^{\beta} \mathbf{y}=\mathbf{F}(\mathbf{y}(t))$, as derive and response systems, are coupled according to the method of Pecora and Carroll [3] with $\alpha=\beta$. Then the stability analysis of linearized system $\mathbf{D}^{\alpha} \mathbf{e}=\mathbf{A e}$, which is found by the difference between two above systems, yields a good criteria for the stability of the phase synchronization between the derive and response systems. More precisely, in the case of $\alpha=\beta=1$, it is clear from linear stability theory in dynamical systems that the stability type of the zero equilibrium in $\mathbf{D}^{\alpha} \mathbf{e}=$ Ae reflects the stability type of the synchronization between the two chaotic systems and depends upon the signs of the real parts of the eigenvalues $\mathbf{A}$ [12]. Phase synchronization also occurs if $\mathbf{A}$ does not have full rank, that is, if $\mathbf{A}$ has at least one zero eigenvalue. For the case of $\alpha$ and $\beta$ less than 1, we can use well-known theorem of Matignon [13]. Because in the case of phase synchronization the error $\mathbf{e}(t)$ converges to a constant or remains bounded by a constant, we may modify Matignon's theorem to the following. 


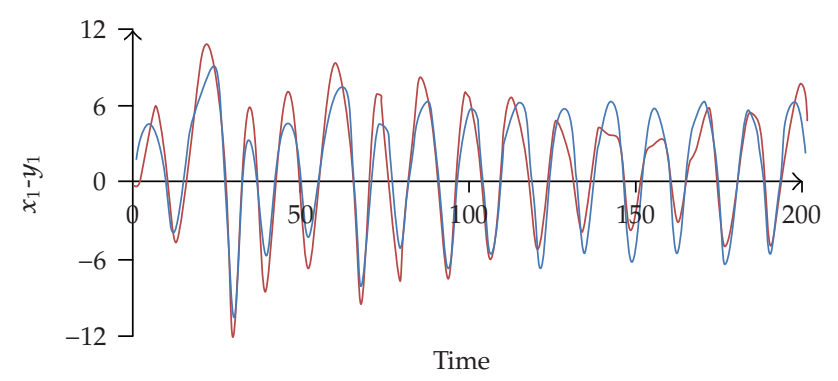

(a)

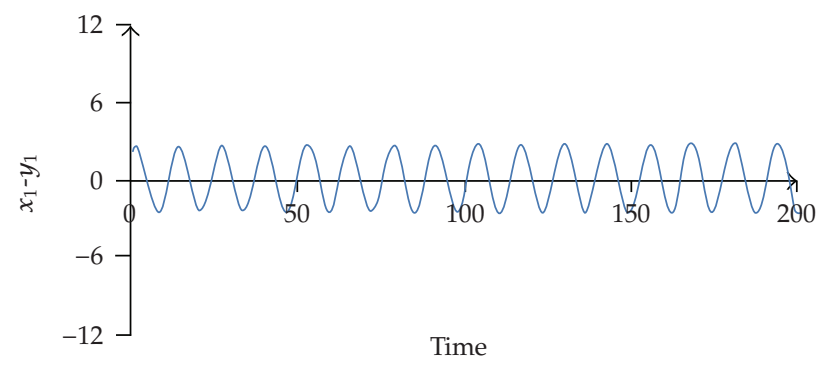

(b)

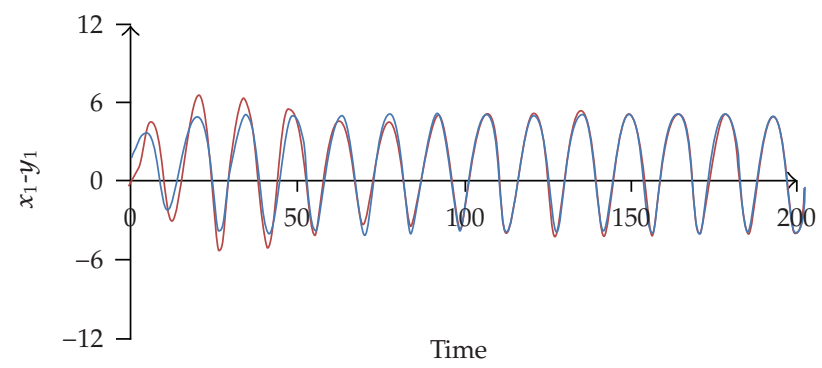

(c)

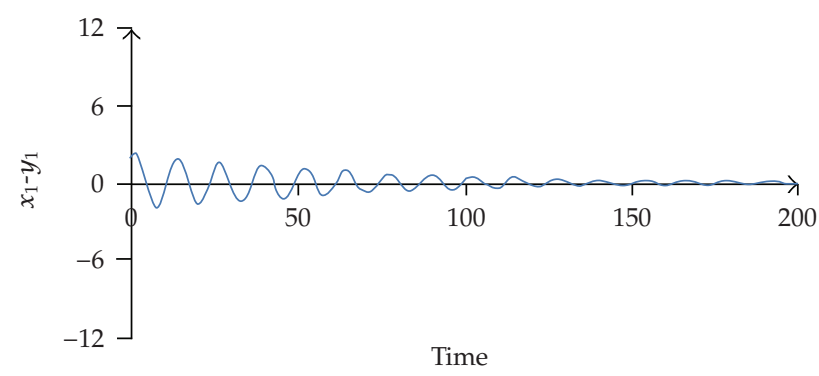

- Series 1

- Series 2

(d)

Figure 3: Phase synchronization for coupled chaotic Sprott-L presented by FDEs. (a) shows the solution $x_{1}$ as derive (Series 1 ) and $y_{1}$ as response (Series 2 ) for $\alpha=\beta=1$, and (b) shows the oscillatory difference between derive and response. (c) shows the solution $x_{1}$ as derive (Series 1) and $y_{1}$ as response (Series 2) for $\alpha=\beta=0.96$, and (d) shows the difference between derive and response that is converging to zero in an oscillatory fashion. 


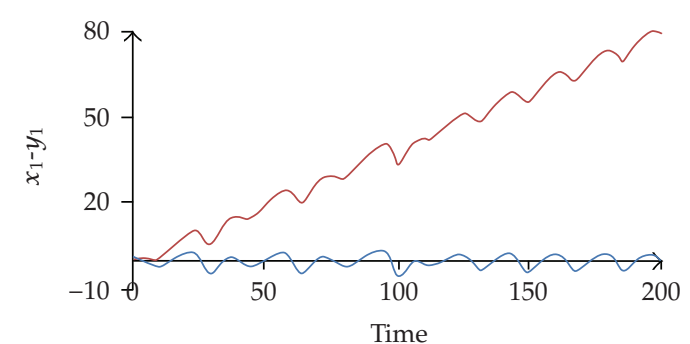

(a)

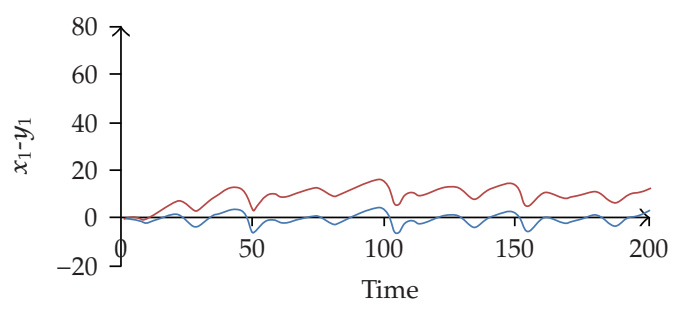

(c)

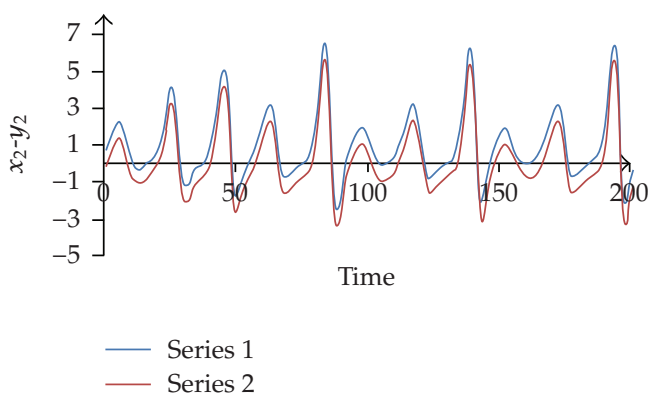

(e)

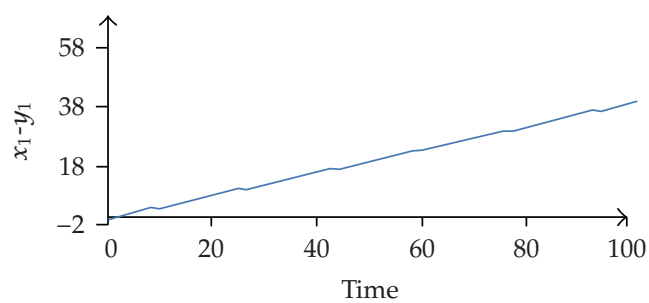

(b)

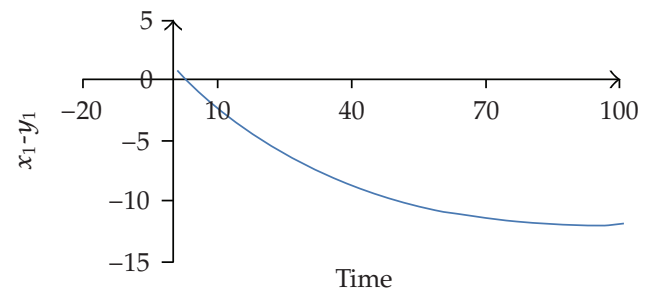

(d)

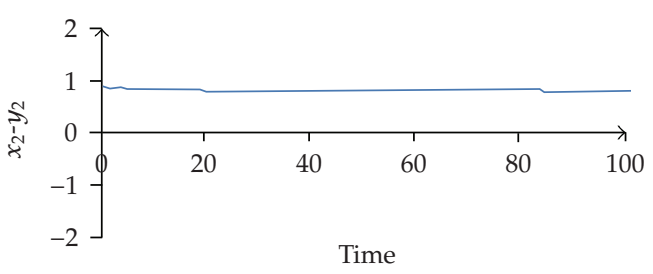

(f)

Figure 4: Phase synchronization for coupled chaotic Sprott-R presented by FDEs. (a) shows the solution $x_{1}$ as derive (Series 1) and $y_{1}$ as response (Series 2) for $\alpha=\beta=1$, and (b) shows the time dependent difference between derive and response. (c) shows the solution $x_{1}$ as derive (Series 1 ) and $y_{1}$ as response (Series 2) for $\alpha=\beta=0.96$, and (d) shows the time dependent difference between derive and response. (e) shows the solution $x_{2}$ as derive (Series 1 ) and $y_{2}$ as response (Series 2) for $\alpha=\beta=0.96$, and (f) shows the constant difference between derive and response.

Theorem 3.1. Define $\mathbf{E}(t)=\mathbf{e}(t)-\mathbf{c}$. Then the linear system of fractional differential equations $\mathbf{E}^{\alpha}(t)=\mathbf{A E}(t)$ is asymptotically stable if and only if $|\arg \operatorname{spc}(\mathbf{A})|>\alpha \pi / 2$. In this case the vector $\mathbf{e}(t)$ converges to $\mathbf{c}$ at the rate $t^{-\alpha}$.

Now it is easy to see that $|\arg \operatorname{spc}(\mathbf{A})|$ for the coupled FDEs of Sprott-S and Sprott-L systems, in Examples 2.1 and 2.3, are 4 and $\sqrt{3.9}$, respectively. By using this modified theorem of Matignon, if there is phase synchronization in these two coupled chaotic systems, then it is convergent for any $\alpha$ and $\beta$ less than one. However, for the coupled FDEs of Sprott-C and Sprott-R systems in Examples 2.2 and 2.4, on which $|\arg \operatorname{spc}(\mathbf{A})|$ is one, this modified theorem does not apply. However, in this case we may use the following convergence criterion which is discussed by Zhang and Sun [14] and Erjaee [12]. 


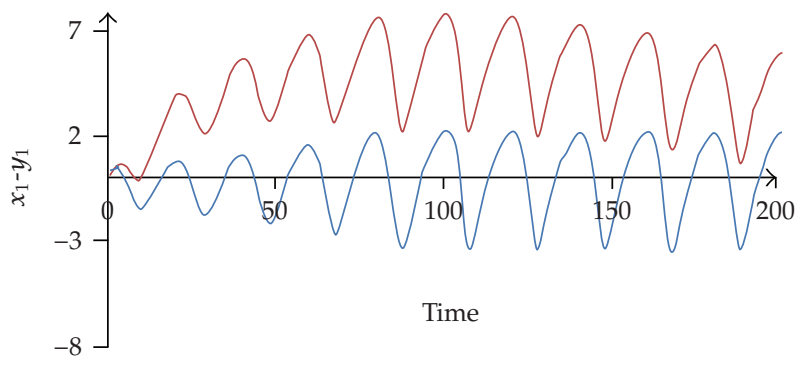

(a)

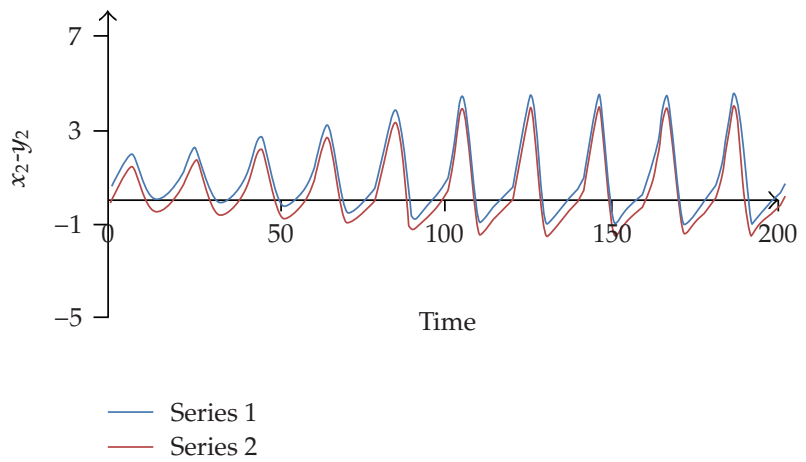

(b)

Figure 5: Solutions of coupled Sprott-R presented by FDEs which illustrate the chaotic behavior turned into periodic solutions for $\alpha=\beta=0.93$. (a) shows the solution $x_{1}$ as derive (Series 1 ) and $y_{1}$ as response (Series 2), and (b) shows the solution $x_{2}$ as derive (Series 1 ) and $y_{2}$ as response (Series 2).

First we define matrix measure of $\mathbf{A} \in \mathbf{R}^{n \times n}$ as $\mu(\mathbf{A})=\lim _{\varepsilon \rightarrow 0}((\|\mathbf{I}-\varepsilon \mathbf{A}(t)\|-1) / \varepsilon)$, where $\mathbf{I}$ is the $n \times n$ identity matrix and $\|\cdot\|$ is any well-known matrix norm, such as one, two, infinity, or the $\omega$-norm defined by $\|\mathbf{A}\|_{\omega}=\max _{j} \sum_{i=0}^{n}\left(\omega_{i} / \omega_{j}\right)\left|a_{i j}\right|$ with $\omega_{i}>0$. Now, different matrix measure can be defined as

$$
\begin{aligned}
\mu_{1}(\mathbf{A}) & =\max _{j}\left\{a_{j j}+\sum_{i=1, i, \neq j}^{n}\left|a_{i j}\right|\right\}, \\
\mu_{2}(\mathbf{A}) & =\frac{1}{2} \lambda_{\max }\left(\mathbf{A}^{T}+\mathbf{A}\right), \\
\text { or } \quad \mu_{\omega}(\mathbf{A}) & =\max _{j}\left\{a_{j j}+\sum_{i=1, i, \neq j}^{n} \frac{\omega_{i}}{\omega_{j}}\left|a_{i j}\right|\right\},
\end{aligned}
$$

with $\omega_{i}>0$. The following theorem shows that under some conditions the phase synchronization in Sprott- $\mathrm{C}$ or Sprott- $\mathrm{R}$ is globally asymptotically stable around a constant vector $\mathbf{c}$ on which $\mathbf{e}(t)=\mathbf{x}(t)-\mathbf{y}(t)=\mathbf{c}$. 
Theorem 3.2. Suppose that $\lim _{t \rightarrow+\infty} \int_{t_{0}}^{t} \mu(\mathbf{A}(t)) d t=-\infty$ for some matrix measure $\mu$ and $t_{0}>0$. Then the system $\mathbf{\mathbf { e }}=\mathbf{A e}$ is globally asymptotically stable around a constant vector $\mathbf{c}$. Consequently there is phase synchronization between derive and response systems, which is globally asymptotically stable.

For the proof, see [12]. Now the matrix measure $\mu_{1}(\mathbf{A})$ in coupled Sprott-C system in Example 2.2 is -1 , while the matrix measure $\mu_{2}(\mathbf{A})$ in the coupled Sprott- $R$ system in Example 2.4 is -2 . Consequently by Theorem 3.2, these two negative matrix measures guarantee the global asymptotical stability of the phase synchronizations in the coupled Sprott-C and Sprott-R systems presented by FDEs, whenever existing.

\section{Conclusion}

We have discussed the existence of phase synchronization in four different Sprott systems presented by FDEs. Although the chaos synchronization broadly exists in the chaotic systems, for example, refer to [15-17], phase synchronization is rear in the chaotic systems, and whenever it does exit, it is very sensitive to the fractional order of the derivatives in both derive and response systems. Since in this article we chose the two identical systems in our coupling using the method of Pecora and Carroll, we restricted ourselves to the choice of two identical values for $\alpha$ and $\beta$ as the orders of derivatives in the derive and response systems. Otherwise the phase synchronization would occur for smaller values than the ones that chose here. For example, during our investigation, we saw that phase synchronization occurs for $\alpha=0.9$ and $\beta=0.5$ or for even smaller values in all the above four examples. However, these systems would not be identical.

\section{Acknowledgment}

This work is supported by Qatar National Research Fund under the Grant number NPRP 08-056-1-014.

\section{References}

[1] J. W. Shuai, K. W. Wong, and L. M. Cheng, "Synchronization of spatiotemporal chaos with positive conditional Lyapunov exponents," Physical Review E, vol. 56, no. 2, pp. 2272-2275, 1997.

[2] G. H. Erjaee, M. H. Atabakzade, and L. M. Saha, "Interesting synchronization-like behavior," International Journal of Bifurcation and Chaos, vol. 14, no. 4, pp. 1447-1453, 2004.

[3] L. M. Pecora and T. L. Carroll, "Synchronization in chaotic systems," Physical Review Letters, vol. 64, no. 8, pp. 821-824, 1990.

[4] J. H. Peng, E. J. Ding, M. Ding, and W. Yang, "Synchronizing hyperchaos with a scalar transmitted signal," Physical Review Letters, vol. 76, no. 6, pp. 904-907, 1996.

[5] A. Tamaševicius, A. Cenys, A. Namajunas, and G. Mykolaitis, "Synchronising hyperchaos in infinitedimensional dynamical systems," Chaos, Solitons E Fractals, vol. 9, no. 8, pp. 1403-1408, 1998.

[6] C. K. Duan and S. S. Yang, "Synchronizing hyperchaos with a scalar signal by parameter controlling," Physics Letters A, vol. 229, no. 3, pp. 151-155, 1997.

[7] J. C. Sprott, "Some simple chaotic flows," Physical Review E, vol. 50, no. 2, pp. R647-R650, 1994.

[8] T. L. Carroll and L. M. Pecora, "Driving systems with chaotic signals," Physical Review A, vol. 44, no. 4, pp. 2374-2383, 1991.

[9] I. Podlubny, Fractional Differential Equations, vol. 198 of Mathematics in Science and Engineering, Academic Press, New York, NY, USA, 1999.

[10] K. B. Oldham and J. Spanier, The Fractional Calculus, Academic Press, New York, NY, USA, 1974. 
[11] J. M. González-Miranda, "Chaotic systems with a null conditional Lyapunov exponent under nonlinear driving," Physical Review E, vol. 53, no. 1, pp. R5-R8, 1996.

[12] G. H. Erjaee, "On analytical justification of phase synchronization in different chaotic systems," Chaos, Solitons E Fractals, vol. 39, no. 3, pp. 1195-1202, 2009.

[13] D. Matignon, "Stability results of fractional differential equations with applications to control processing," in Proceeding of the IMACS-IEEE Multiconference on Computational Engineering in Systems Applications (CESA '96), vol. 963, Lille, France, July 1996.

[14] Y. Zhang and J. Sun, "Chaotic synchronization and anti-synchronization based on suitable separation," Physics Letters A, vol. 330, no. 6, pp. 442-447, 2004.

[15] C. P. Li and G. J. Peng, "Chaos in Chen's system with a fractional order," Chaos, Solitons E Fractals, vol. 22, no. 2, pp. 443-450, 2004.

[16] W. H. Deng and C. P. Li, "Chaos synchronization of the fractional Lü system,” Physica A, vol. 353, no. 1-4, pp. 61-72, 2005.

[17] C. P. Li and W. H. Deng, "Chaos synchronization of fractional-order differential systems," International Journal of Modern Physics B, vol. 20, no. 7, pp. 791-803, 2006. 


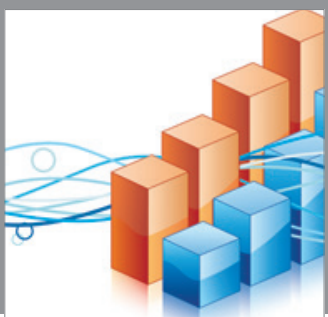

Advances in

Operations Research

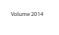

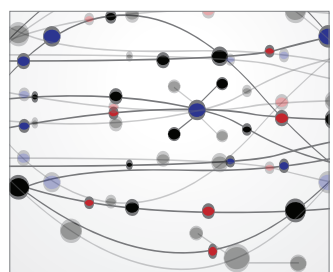

\section{The Scientific} World Journal
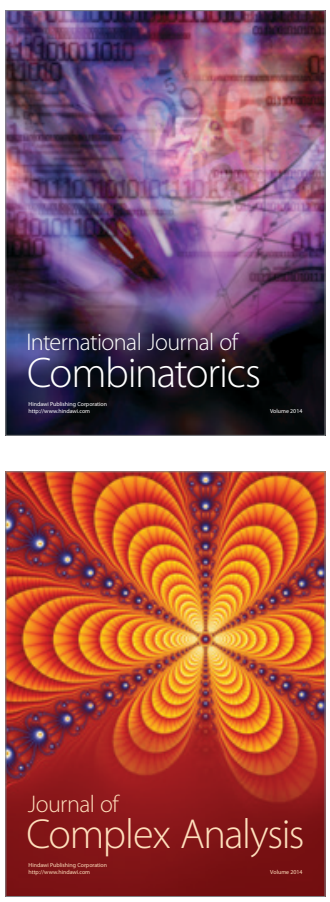

International Journal of

Mathematics and

Mathematical

Sciences
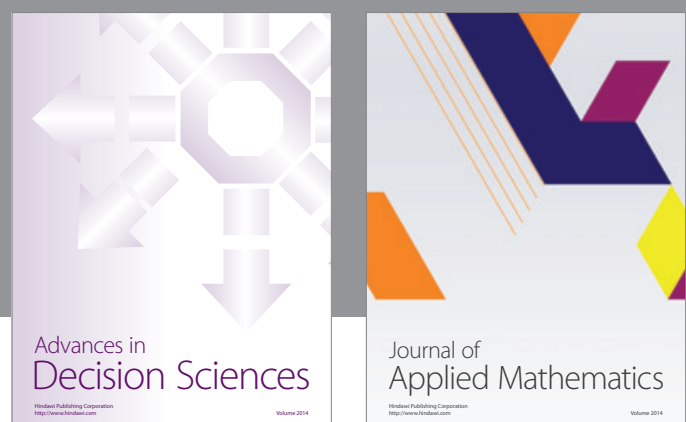

Journal of

Applied Mathematics
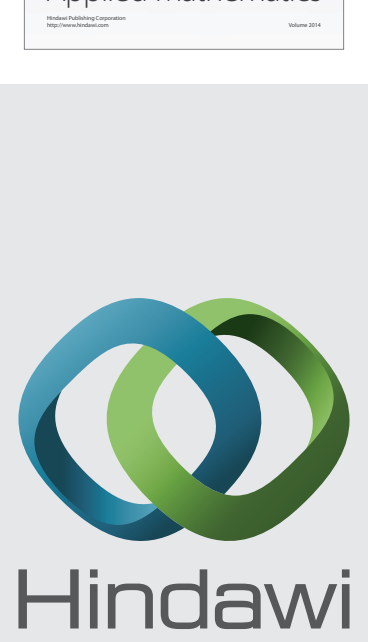

Submit your manuscripts at http://www.hindawi.com
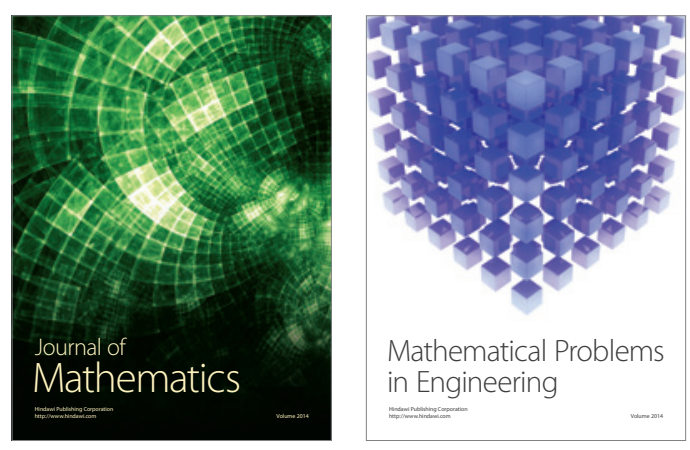

Mathematical Problems in Engineering
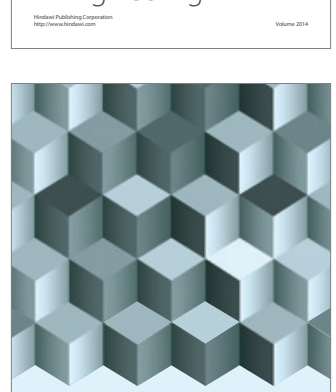

Journal of

Function Spaces
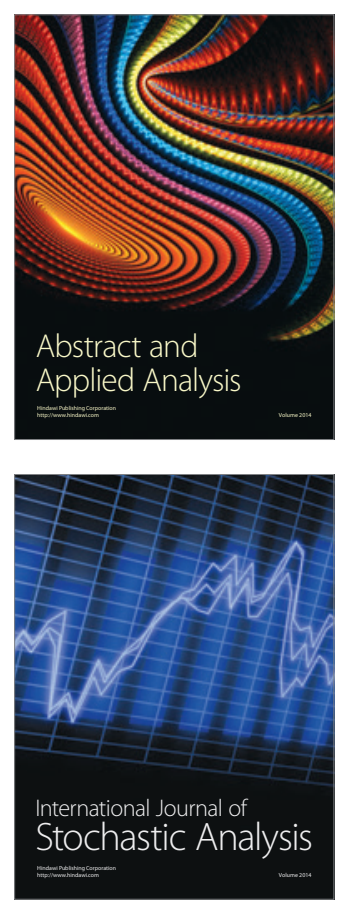

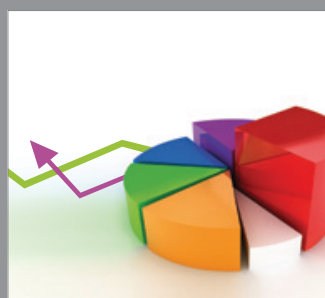

ournal of

Probability and Statistics

Promensencen
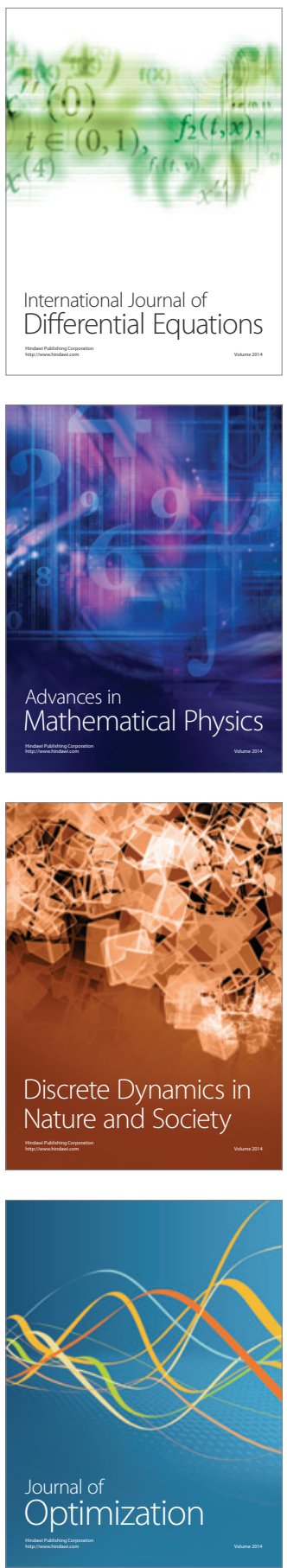